\title{
DEGENERATE ELLIPTIC OPERATORS AS REGULARIZERS
}

\author{
R. N. PEDERSON
}

\begin{abstract}
The spaces $\mathscr{K}_{m k}$, introduced in the Nehari Volume of Journal d'Analyse Mathématique, for nonnegative integer values of $m$ and arbitrary real values of $k$ are extended to negative values of $m$. The extension is consistent with the equivalence $\left\|\zeta^{j} u\right\|_{m, k} \sim\|u\|_{m, k-j}$, the inequality $\left\|D^{\alpha} u\right\|_{m, k} \leqslant$ const $\|u\|_{m+|\alpha|, k+|\alpha|}$, and the generalized Cauchy-Schwarz inequality $|\langle u, v\rangle| \leqslant\|u\|_{m, k}\|v\|_{-m,-k}$. (Here $\langle u, v\rangle$ is the $L_{2}$ scalar product.) There exists a second order degenerate elliptic operator which maps $\mathcal{K}_{m, k} 1-1$ onto $\mathcal{K}_{m-2, k}$. These facts are used to simplify proof of regularity theorems for elliptic and hyperbolic problems and to give new results concerning growth rates at the boundary for the coefficients of the operator and the forcing function. (See Notices Amer. Math. Soc. 28 (1981), 226.)
\end{abstract}

1. Introduction. It is the purpose of this paper to show how the equivalent norms for the Sobolev spaces introduced by the author in [11] can be used in conjunction with a special class of degenerate second order elliptic operators to study the regularity of solutions of other differential equations. We shall assume throughout the present paper that $\Omega$ is a compact domain of class $e^{j}, j \geqslant 1$. As in [11] we use the fact that there exists a function $\zeta \in e^{j}(\bar{\Omega})$ such that

$$
\zeta>0, \quad x \in \Omega, \quad \zeta=0, \quad \operatorname{grad} \zeta \neq 0, \quad x \in \partial \Omega .
$$

and that when $m \leqslant j$ the completion $\mathcal{H}_{k}^{m}$ of $\bigodot_{0}^{\infty}(\Omega)$ for any real $k$ under the norm

$$
\|U\|_{m, k}^{2}=\sum_{|\alpha| \leqslant m} \int_{\Omega} \zeta^{2(|\alpha|-k)}\left(D^{\alpha} U(x)\right)^{2} d x
$$

is the same as the completion of the $\bigodot^{\infty}$ functions for which the norm (1.2) is finite.

From this point on we shall assume that $\Omega$ is a domain of class $e^{j}, j \geqslant 2$, and that $\left(Q_{j k}(x)\right)$ is a real positive definite second order covariant tensor of class $\mathcal{C}^{j}$ and inverse $\left(\mathbb{Q}^{j k}(x)\right)$ and $a=\operatorname{det}\left(Q_{i j}\right)$, and shall regard $\Omega$ as a Riemannian manifold with metric tensor $\left(Q_{j k}\right)$. For a function $\zeta$ satisfying (1.1) we shall study the degenerate elliptic operator

$$
E_{K} U=-\frac{1}{\sqrt{a}} \frac{\partial U}{\partial x_{j}} \zeta^{2} \sqrt{a} \Theta^{j k} \frac{\partial U}{\partial x_{k}}+K U, \quad K>0 .
$$

If we write $E_{K} U$ in the form

$$
E_{K} U=-\frac{\zeta^{2}}{\sqrt{a}} \frac{\partial}{\partial x_{j}} \sqrt{a} \Theta^{j k} \frac{\partial u}{\partial x_{k}}-2 \zeta \frac{\partial \zeta}{\partial x^{j}} \mathbb{Q}^{j k} \frac{\partial u}{\partial x_{k}}+K u,
$$

Received by the editors September 2, 1982; presented to the Society, May 15, 1981 at Duquesne University.

1980 Mathematics Subject Classification. Primary 35A99; Secondary 34A99.

(C)1983 American Mathematical Society $0002-9947 / 83 \$ 1.00+\$ .25$ per page 
the first term on the right is a scalar multiple of the Laplace-Beltrami operator corresponding to the metric $\left(Q_{j k}\right)$. Under a change of coordinates $x \rightarrow y$, the second term on the right transforms by the rule

$$
\zeta \frac{\partial \zeta}{\partial x^{j}} \mathbb{Q}^{j k} \frac{\partial u}{\partial x_{k}}=\zeta \frac{\partial \zeta}{\partial y^{\nu}} \frac{\partial y^{\nu}}{\partial x^{j}} \mathbb{Q}^{j k} \frac{\partial y^{\mu}}{\partial x^{k}} \frac{\partial u}{\partial y^{\mu}}
$$

and, since $\mathbb{Q}^{j k}$ is a contravariant tensor, it follows that the operator $E_{K}$ is invariantly defined although this fact is a convenience for our proofs and not a necessity.

Since the operator (1.3) is completely degenerate on the boundary, we have uniqueness of classical solutions of

$$
E_{K} u=f \in e^{\infty}(\bar{\Omega})
$$

with no boundary conditions at all. One would therefore expect that solutions of a differential equation

$$
P u=f \in e^{\infty},
$$

which are unique under the boundary condition

$$
B u=0, \quad x \in \partial \Omega,
$$

would also be the unique solution of

$$
E_{K}^{\nu} P u=E_{K}^{\nu} f, \quad B u=0, \quad x \in \partial \Omega .
$$

Equation (1.9), being of higher order at interior points of $\Omega$, should then possess more regularity a priori than those of (1.7)-(1.8). The above statements are, of course, meaningless in the absence of a characterization of the solution spaces. It is to this study that the present paper is addressed. The introduction of the degenerate elliptic operator (1.3) is motivated by Friedrichs' Clipped Laplacian [3]. The introduction of the spaces $\mathcal{K}_{m, k}$, together with the equivalence of $\mathcal{H}_{m m}$ and the classical Sobolev space $\mathcal{H}_{m}$, proved by the author in [11], makes possible more systematic use. In particular, our theorems of $\$ 5$ show that, although solutions of $E_{K} u=f \in \mathcal{C}_{0}^{\infty}$ do not necessarily vanish on the boundary, for $K$ sufficiently large they do vanish to any preassigned order. Moreover, if the boundary is sufficiently smooth, the same is true of derivatives of solutions. Hence, solutions of $E_{K} u=f \in \bigodot_{0}^{\infty}$ can be used as test functions.

2. The Lax duality lemma. The standard theorems on negative norm spaces do not apply directly to the norm $\|U\|_{m, k}$. The following theorem shows that the original construction of Lax [6] applies to a wide class of Hilbert spaces other than the classical Sobolev spaces.

TheOREM 2.1. Let $\mathcal{H}$ be a complex Hilbert space with inner product ( , ), V a complex vector space and $\langle$,$\rangle a bilinear form from \mathcal{H} \times V$ to the complex numbers such that

$$
\text { for each } v \in V,\langle\cdot, v\rangle \text { is a bounded linear functional on } \mathcal{H} \text {, }
$$

and

$$
\text { if }\langle u, v\rangle=0 \text { for all } u \in \mathcal{H} \text {, then } v=0 \text {, }
$$

$$
\text { if }\langle u, v\rangle=0 \text { for all } v \in V \text {, then } u=0 \text {. }
$$


Then there exists a Hilbert space $\mathcal{H}^{-} \supset V$ which is isometrically isomorphic to $\mathcal{H}$ and an extension $\langle\langle\rangle$,$\rangle of \langle$,$\rangle to \mathcal{H} \times \mathcal{H}^{-}$such that the generalized Cauchy-Schwarz inequality

$$
|\langle\langle u, v\rangle\rangle| \leqslant\|u\|\|v\|^{-}, \quad u \in \mathcal{H}, v \in \mathcal{H}^{-},
$$

is valid. The norms \|\| and \|\|$^{-}$bear the reciprocal relations

$$
\|v\|^{-}=\sup _{u \in \mathcal{H}} \frac{\mid\langle\langle u, v\rangle\rangle}{\|u\|}, \quad v \in \mathcal{H}^{-},
$$

and

$$
\|u\|=\sup _{v \in \mathcal{H}} \frac{\mid\langle\langle u, v\rangle\rangle}{\|v\|^{-}}, \quad u \in \mathcal{H}
$$

Proof. As a consequence of $(2.1)$ for each $v \in V$, there exists a $T v \in \mathcal{H}$ such that

$$
\langle u, v\rangle=(u, T v) \text {. }
$$

$T$ is evidently a linear operator. Define

$$
\|v\|^{-}=\sup _{u \in \mathcal{X}} \frac{|\langle u, v\rangle|}{\|u\|} .
$$

It follows from (2.7) and (2.8) that

$$
|v|^{-}=\|T v\|
$$

hence the mapping $T$ is an isometry. It follows from (2.2) that $\| \mathrm{I}^{-}$is positive. The polarization identity for $\|v\|^{-}$follows from (2.7) and (2.8). Hence $U$ is a pre-Hilbert space with the inner product $(,)^{-}$induced by \|\|$^{-}$.

We now define $\mathcal{H}^{-}$to be the completion of $V$ with respect to $\|v\|^{-}$; hence $V$ is dense in $\mathcal{H}^{-}$. Since (2.3) implies that $\overline{T V}=\mathcal{H}$, it follows that

$$
\|u\|=\sup _{v \in U} \frac{|\langle u, v\rangle|}{\|v\|^{-}},
$$

which in turn implies the generalized Cauchy-Schwarz inequality on $\mathcal{H} \times V$,

$$
|\langle u, v\rangle| \leqslant\|u\|\|v\|^{-}, \quad u \in \mathcal{H}, v \in V .
$$

By the Metric Completion Theorem, $\mathcal{H}^{-}$is a set of equivalence classes $\left\{v_{n}\right\}$ of Cauchy sequences in $V$. Hence, we may define the extension $\langle\langle\rangle$,$\rangle by$

$$
\left\langle\left\langle u,\left\{v_{n}\right\}\right\rangle\right\rangle=\lim _{n \rightarrow \infty}\left\langle u, v_{n}\right\rangle .
$$

The limit (2.12) exists since, by (2.11),

$$
\left|\left\langle u, v_{n}\right\rangle-\left\langle u, v_{m}\right\rangle\right| \leqslant\|u\|\left\|v_{n}-v_{m}\right\|^{-} \rightarrow 0 \quad \text { as } m, n \rightarrow \infty .
$$

That the limit is unique independently of the representative of the equivalence class follows from standard arguments. It follows from (2.9) that $T$ has an extension which is 1-1 and onto. Hence $\mathcal{H}$ and $\mathcal{H}^{-}$are isometrically isomorphic. This completes the proof. 
By interchanging the roles of $\mathcal{K}$ and $\mathcal{H}^{-}$we see that there exists an isometric isomorphism $S$ of $\mathcal{H}$ onto $\mathcal{H}^{-}$such that

$$
(S u, v)^{-}=\langle\langle u, v\rangle\rangle=(u, T v)
$$

for all $u \in \mathcal{H}, v \in \mathcal{H}^{-}$. In particular, this allows us to represent linear functions on either $\mathcal{H}^{-}$or $\mathcal{H}$ in terms of the bilinear form $\langle\langle\rangle$,$\rangle . When no serious confusion can$ result, we shall identify $\langle$,$\rangle with its extension.$

3. The Lax duals of the spaces $\mathcal{H}_{m, k}$. The norms

$$
\|u\|_{m, k}^{2}=\sum_{|\alpha| \leqslant m} \int_{\Omega} \zeta^{2(|\alpha|-k)}\left|D^{\alpha} u(x)\right|^{2} d x
$$

were defined in [11] for $m$ a nonnegative integer and $k$ an arbitrary real number. They were shown to have the same completion for the $\bigcup^{\infty}$ functions with finite norm as for the $e_{0}^{\infty}$ functions. If we define the completion of $C_{0}^{\infty}(\Omega)$, with respect to $\|u\|_{m, k}$, to be $\mathcal{H}_{m, k}(\Omega)$, the results of $\S 2$, relative to the bilinear form

$$
\langle u, \varphi\rangle=\int_{\Omega} u(x) \varphi(x) d x, \quad u \in \mathcal{H}_{m, k}, \varphi \in \bigodot_{0}^{\infty},
$$

yield the Lax dual space $\mathcal{H}_{m, k}^{-}$with inner product induced by the norm

$$
\|u\|_{m, k}^{-}=\sup _{\varphi \in \mathcal{C}_{0}^{\infty}(\Omega)} \frac{|\langle u, \varphi\rangle|}{\|\varphi\|_{m, k}},
$$

the reciprocal relation

$$
\|u\|_{m, k}=\sup _{\varphi \in \mathcal{H}_{m, k}^{-}} \frac{|\langle\varphi, u\rangle|}{\|\varphi\|_{m, k}^{-}}=\sup _{\varphi \in \mathcal{C}_{0}^{\infty}} \frac{|\langle\varphi, u\rangle|}{\|\varphi\|_{m, k}^{-}},
$$

and the generalized Cauchy-Schwarz inequality

$$
|\langle u, \varphi\rangle| \leqslant\|u\|_{m, k}\|\varphi\|_{m, k}^{-}, \quad u \in \mathcal{H}_{m, k}, \varphi \in \mathcal{H}_{m, k}^{-} .
$$

We shall show in this section that it makes sense to interpret $\mathcal{H}_{m, k}^{-}$as $\mathcal{H}_{-m,-k}$. This interpretation is a consequence of the following useful equivalences and inequalities.

THEOREM 3.1. If $m$ is a nonnegative real number and $k$ is an arbitrary real number, then, for any real $j$,

$$
\left\|\zeta^{j} u\right\|_{m, k} \sim\|u\|_{m, k-j},
$$

and if $D$ represents a generic first derivative, then

$$
\|D u\|_{m, k} \leqslant\|u\|_{m+1, k+1} .
$$

Proof. It follows from Leibniz's rule that

$$
D^{\alpha}\left(\zeta^{j} u\right)=\sum_{\beta \leqslant \alpha}\left(\begin{array}{l}
\alpha \\
\beta
\end{array}\right) D^{\alpha-\beta} \zeta^{j} D^{\beta} u .
$$

Since there exists a constant $e_{j, \gamma}$ such that

$$
\left|D^{\gamma} \zeta^{j}\right| \leqslant e_{j, \gamma} \zeta^{j-|\gamma|},
$$


it follows that there exists a constant $D_{m, j}$ such that, for $|\alpha| \leqslant m$,

$$
D^{\alpha}\left(\zeta^{j} u\right)\left|\leqslant D_{m, j} \sum_{|\beta| \leqslant|\alpha|} \zeta^{(j+|\beta|-|\alpha|)}\right| D^{\beta} u \mid
$$

It follows by substituting (3.9) into (3.1) that

$$
\left\|\zeta^{j} u\right\|_{m, k}^{2} \leqslant \text { const }\|u\|_{m, k-j}
$$

The equivalence (3.6) is established by replacing $u$ by $\zeta^{-j} u$ in (3.10) and then applying (3.10) with $j$ replaced by $-j$ and $k$ replaced by $k-j$. The inequality (3.7) follows immediately by substituting $D u$ for $u$ in (3.1).

We next show that Theorem 3.1 can be consistently extended to negative norms $\|u\|_{m, k}^{-}$.

THEOREM 3.2. If $m$ is a nonnegative integer and $k$ a real number then, for any real $j$,

$$
\left\|\zeta^{j} u\right\|_{m, k}^{-} \sim\|u\|_{m, k+j}^{-}
$$

Proof. We first observe that if $\psi$ is a fixed function in $e^{\infty}(\Omega)$ which does not vanish at interior points of $\Omega$, then (3.3) may be replaced by

$$
\|u\|_{m, k}^{-}=\sup _{\varphi \in \mathcal{C}_{0}^{\infty}} \frac{|\langle u, \psi \varphi\rangle|}{\|\psi \varphi\|_{m, k}} .
$$

It follows from (3.3) that

$$
\left\|\zeta^{j} u\right\|_{m, k}^{-}=\sup _{\varphi \in \mathcal{C}_{0}^{\infty}(\Omega)} \frac{\left|\left\langle\zeta^{j} u, \varphi\right\rangle\right|}{\|\varphi\|_{m, k}}=\sup _{\varphi \in \mathcal{C}_{0}^{\infty}(\Omega)} \frac{\left|\left\langle u, \zeta^{j} \varphi\right\rangle\right|}{\left\|\zeta^{-j}\left(\zeta^{j} \varphi\right)\right\|_{m, k}} .
$$

Hence, by (3.12),

$$
\left\|\zeta^{j} u\right\|_{m, k}^{-}=\sup _{\varphi \in \mathcal{C}_{0}^{\infty}(\Omega)} \frac{|\langle u, \varphi\rangle|}{\left\|\zeta^{-j} \varphi\right\|_{m, k}} .
$$

It now follows from (3.6) and (3.14) that (3.11) is valid.

We are now in a position to define $\mathcal{H}_{m, k}^{-}$to be equal to $\mathcal{H}_{-m,-k}$, and to retain the validity of (3.6) for negative integers $m$.

THEOREM 3.3. The definition

$$
\|u\|_{m, k}^{-}=\|u\|_{-m,-k}
$$

is consistent with the validity of (3.6) for negative $m$.

Proof. The notation (3.15) when applied to (3.11) gives

$$
\left\|\zeta^{j} u\right\|_{-m,-k} \sim\|u\|_{-m,-k,-j}
$$

which is just (3.6) with $m$ and $k$ replaced by their negatives.

The above theorem makes it possible also to extend (3.7) to negative values of $m$.

THEOREM 3.4. The definition (3.15) is consistent with the validity of (3.7) for negative $m$. 
Proof. We first consider the case where $m \geqslant 1$. Then, by (3.3),

$$
\|D u\|_{m, k}^{-}=\sup _{\varphi \in \mathcal{C}_{0}^{\infty}} \frac{|\langle D u, \varphi\rangle|}{\|\varphi\|_{m, k}}=\sup _{\varphi \in \mathcal{C}_{0}^{\infty}} \frac{|\langle u, D \varphi\rangle|}{\|\varphi\|_{m, k}} .
$$

The generalized Cauchy-Schwarz inequality (3.5) implies that

$$
\begin{aligned}
|\langle u, D \varphi\rangle| & \leqslant\|u\|_{m-1, k-1}^{-}\|D \varphi\|_{m-1, k-1} \\
& \leqslant\|u\|_{m-1, k-1}^{-}\|\varphi\|_{m, k}
\end{aligned}
$$

as a consequence of (3.7). It follows from (3.17) and (3.18) that

$$
\|D u\|_{m, k}^{-} \leqslant\|u\|_{m-1, k-1}^{-} .
$$

The definition (3.15) applied to (3.19) now gives

$$
\|D u\|_{-m,-k} \leqslant\|u\|_{1-m, 1-k}
$$

when $m \geqslant 1$. But (3.20) is just (3.7) with $m$ and $k$ replaced by their negatives. When $m=0,(3.15)$ gives

$$
\|D u\|_{0, k}^{-}=\|D u\|_{0,-k} \leqslant\|u\|_{1,1-k}
$$

as a consequence of (3.1). This completes the proof of Theorem 3.4.

At times it is convenient to have the following equivalent formulation of the norm $\|u\|_{m, k}$.

THEOREM 3.5. For $K$ sufficiently large the norms $\|u\|_{m+2, k}$ and $\left\|E_{K} u\right\|_{m, k}$ are equivalent.

Proof. Consider first the case $m=0, k=0$. It follows from integration by parts that, for $u \in \mathcal{C}_{0}^{\infty}(\Omega)$,

$$
\left\|E_{K} u\right\|_{0,0}^{2} \geqslant \int_{\Omega} \zeta^{4} \mathscr{Q}_{i j} \mathscr{Q}_{k e} \frac{\partial^{2} u}{\partial x_{i} \partial x_{k}} \frac{\partial^{2} u}{\partial x_{j} \partial x_{e}} d x-\mathcal{C}\|u\|_{1,0}^{2}+K^{2}\|u\|_{0,0}^{2} .
$$

It was observed by Oleinik [9] and Mizohata [7] that

$$
\mathbb{Q}_{i j} \mathbb{Q}_{k e} \frac{\partial^{2} u}{\partial x_{i} \partial x_{k}} \frac{\partial^{2} u}{\partial x_{i} \partial x_{e}} \geqslant \varepsilon \sum_{|\alpha|=2}\left|D^{\alpha}\right|^{2}, \quad \varepsilon>0 .
$$

It now follows from (3.22), (3.23) and the interpolation inequalities that

$$
\left\|E_{K} u\right\|_{0,0} \geqslant\|u\|_{2,0} \text {. }
$$

The opposite inequality is trivial. Hence $\|u\|_{2,0}$ and $\left\|E_{K} u\right\|_{0,0}$ are equivalent norms. It follows from Theorem 3.1 and (3.24) that $\|u\|_{m+2, k} \sim\left\|E_{K} u\right\|_{m, k}$ when $m \geqslant 0$. The completion of the proof for $m<0$ will be deferred to the end of $\S 5$.

We shall also find the following lemma useful.

LEMMA 3.6. If $u$ is a solution of $E_{K} u=f$ where $u \in \mathcal{H}_{2,0}$, and for some $\nu>0$, $u \in \mathcal{H}_{1, \nu}, f \in \mathcal{H}_{0, \nu}$, then $u \in \mathcal{H}_{2, \nu}$.

Proof. It follows from the equation that

$$
\int(\zeta+\varepsilon)^{-2 \nu}\left|E_{K} u\right|^{2} d k=\int(\zeta+\varepsilon)^{-2 \nu}|f|^{2} d x
$$


An application of Leibniz's formula then yields

$$
\begin{aligned}
\left\|E_{K}(\zeta+\varepsilon)^{-\nu} u\right\|_{0,0}^{2} \leqslant & \left\|(\zeta+\varepsilon)^{-\nu-1} \zeta^{2} \mid \operatorname{grad} u\right\|_{0,0}^{2} \\
& +\left\|(\zeta+\varepsilon)^{-\nu-2} \zeta^{2} u\right\|_{0,0}^{2}+\left\|(\zeta+\varepsilon)^{-\nu} f\right\|_{0,0}^{2}
\end{aligned}
$$

It follows from (3.26) and Theorem 3.5 that

$$
\begin{aligned}
\left\|(\zeta+\varepsilon)^{-\nu} u\right\|_{2,0} \leqslant \operatorname{const}\{ & \left\|(\zeta+\varepsilon)^{-\nu-1} \zeta^{2}(\operatorname{grad} u)\right\|_{0,0} \\
& \left.+\left\|(\zeta+\varepsilon)^{-\nu-2} \zeta^{2} u\right\|_{0,0}^{2}+\left\|(\zeta+\varepsilon)^{-\nu} f\right\|_{0,0}\right\} .
\end{aligned}
$$

The hypothesis implies that we may replace $\varepsilon$ by zero on the right side of (3.23). The proof then is a consequence of Fatou's Lemma.

4. Existence, uniqueness and regularity for solutions of the regularizing equation. The weak formulation of the assertion that $u$ is a weak solution of (1.6) is that

$$
\int_{\Omega}\left[\zeta^{2} a^{j k}(x) \frac{\partial u}{\partial x^{i}} \frac{\partial \varphi}{\partial x^{k}}+K u \varphi\right] \sqrt{a} d x=\int_{\Omega} f \varphi d x
$$

for all $\varphi \in C_{0}^{\infty}(\Omega)$. Since the left side of (4.1) defines an inner product for the space $\mathcal{H}_{1,0}(\Omega)$ for any $K>0$, it is an immediate consequence of the Riesz Representation Theorem that for each $f \in \mathcal{H}_{-1,0}(\Omega)$ there exists a unique solution $u \in \mathcal{H}_{1,0}(\Omega)$ of (4.1). We shall prove in this section the following regularity theorem.

THEOREM 4.1. If $\Omega$ is a compact domain whose boundary is of class $C^{k}, a^{i j} \in C^{k}$, $k \geqslant 1$, and $f \in \mathcal{H}_{k-2,0}(\Omega)$, then (4.1) has a unique solution $u \in \mathcal{H}_{k, 0}(\Omega)$ for each positive $k$.

Proof. We first use a bootstrap argument to prove that $f \in \mathcal{H}_{0,0}, u \in \mathcal{H}_{1,0}$, implies that $u \in \mathcal{H}_{2,0}(\Omega)$. Let $h \in C^{\infty}(R)$ with $h(t)=0$ for $t<1$ and $h(t)=1$ for $t \geqslant 2$. Let $\Delta^{+}$denote a difference quotient with respect to an arbitrary increment and $\Delta^{-}$the corresponding backward difference. The function $h(n \zeta) u$ then has compact support in $\Omega$ and, since the normal derivative of $\zeta$ is nonzero on $\partial \Omega$, we may, for any $n$, choose an increment such that

$$
\Delta^{-} \Delta^{+} \zeta^{2} h(n \zeta) u
$$

is defined and has compact support in $\Omega$. Since (4.1) extends immediately to $\varphi \in \mathcal{H}_{1,0}(\Omega)$ we may use, as a test function,

$$
\varphi=h(n \zeta) \Delta^{-} \Delta^{+} \zeta^{2} h(n \zeta) u .
$$

After substituting (4.2) into (4.1) and integrating and differencing by parts we obtain

$$
\begin{aligned}
\int \sqrt{a a}^{j k} \frac{\partial}{\partial x_{j}} & \left(\Delta^{+} h(n \zeta) \zeta^{2} u\right) \frac{\partial}{\partial x_{k}} \Delta^{+}\left(h(n \zeta) \zeta^{2} u\right) d x \\
= & \int \Delta^{+}\left\{\sqrt{a} a^{j k} \zeta \frac{\partial \zeta}{\partial x_{k}}\left[n \zeta h^{\prime}(n \zeta)+2 h(n \zeta)\right] u\right\} \frac{\partial}{\partial x_{k}}\left(h(n \zeta) \zeta^{2} u\right) d x \\
& +\int(K u-f) \Delta^{-} \Delta^{+}\left(h(n \zeta) \zeta^{2} u\right) \sqrt{a} d x
\end{aligned}
$$


It follows, from the classical inequality

$$
\left\|\Delta^{ \pm} v\right\|_{L_{2}} \leqslant\|\operatorname{grad} v\|_{L_{2}}
$$

for $v$ with compact support in $\Omega$ and the positive definiteness of $a^{j h}$, that

$$
\begin{aligned}
& \left\|\operatorname{grad} \Delta^{+} h(n \zeta) \zeta^{2} u\right\|_{L_{2}} \\
& \quad \leqslant C\left\{\|K u-f\|_{L_{2}}+\sup \left|n^{2} \zeta^{2} h^{\prime \prime}(n \zeta)+n \zeta h^{\prime}(n \zeta)\right|\|u\|_{1,0}\right\} .
\end{aligned}
$$

Since, for $j \geqslant 1, h^{(j)}(n \zeta)=0$ where $n \zeta>2$, we may substitute the bound $n \zeta \leqslant 2$ and deduce, as a consequence of the facts that $u \in \mathcal{H}_{1,0}, f \in \mathcal{H}_{0,0}$, that the left side of (4.5) is uniformly bounded in $n$. Since a strongly bounded sequence has a weakly convergent subsequence, it follows that each first derivative of $\zeta^{2} u$ has a weak derivative in the direction corresponding to $\Delta^{+}$. By applying this argument to each of the coordinate directions and using the fact that weak derivatives are strong we find that $\zeta^{2} u$ has strong second derivatives. This, together with the fact that $u \in \mathcal{H}_{1,0}$, implies that $u \in \mathcal{H}_{2,0}$. This completes the proof of Theorem 4.1 when $k=2$. The case $k>2$ follows from the special case by using $\zeta^{k} D^{k} \varphi$ as a test function. The following lemma provides the induction step.

LEMMA 4.2. If $u \in \mathcal{H}_{2,0}$ is a weak solution of (1.6) with $f \in \mathcal{H}_{1,0}$, then there exists a function $g \in \mathcal{H}_{0,0}$ such that $\zeta \mathrm{Du}$ is a weak solution of (1.6) with $f$ replaced by $g$ and hence $u \in \mathcal{H}_{3,0}$.

Proof. Choose as test function in (4.1) the function $\zeta D \varphi$ and integrate by parts to obtain

$$
\int_{-\Omega}\left[a^{i j} \frac{\partial}{\partial x^{i}} \zeta D \varphi \frac{\partial \varphi}{\partial x^{j}}+K \zeta D \varphi\right] \sqrt{a} d x=\int_{\Omega} g \varphi \sqrt{a} d x
$$

where $g$ is a linear combination with bounded coefficients of

$$
\zeta^{i} D^{i} f, \quad|i| \leqslant 1 \quad \text { and } \zeta^{i} D^{i} u, \quad|i| \leqslant 2 ;
$$

hence $g \in \mathcal{H}_{0,0}$. It follows from the special case that $\zeta D u \in \mathcal{K}_{0,0}$ and, since $D$ is an arbitrary first derivative, $u \in \mathcal{H}_{3,0}$.

Successive applications of Lemma 4.2 show that if $u \in \mathcal{H}_{2,0}$ is a weak solution of (1.6) with $f \in \mathcal{K}_{\nu-1,0}$, then $\zeta^{\nu-1} D^{\nu-1} u$ is a weak solution of (1.6) for some $g \in \mathcal{K}_{0,0}$. It follows that $u \in \mathcal{H}_{\nu+1,0}$. This completes the proof of Theorem 4.1.

5. Behavior at the boundary of solutions of $E_{K} u=f$. In this section we shall prove that when $f$ is a test function and $K$ is sufficiently large, the solution of $E_{K} u=f$ is also a test function. Up to this point our use of the Laplace-Beltrami operator has been only formal. We now assume that $k \geqslant 2$ so that we have a unique solution of the geodesic equation joining each pair of points which are sufficiently close together. In particular, since $\Omega$ is compact, there exists a $\delta>0$ such that if the distance from $x$ to $\partial \Omega$ is less than $\delta$ there is a unique boundary point which minimizes the distance to $x$. For each $t<\delta$ the set

$$
M_{t}=\{x: \operatorname{dist}(x, \delta \Omega)=t\}
$$


is a smooth $(n-1)$-dimensional manifold without boundary. We may use the coordinates of $M_{0}$ to parametrize $M_{t}$ assigning to $x \in M_{t}$ the unique boundary point which minimizes the geodesic distance to $x$. Thus, if $\left(0, y_{2}, \ldots, y_{n}\right)$ represent a local coordinate system for $M_{0}$ we may parametrize the set

$$
\left\{x \in M_{t}, 0 \leqslant t \leqslant \delta\right\}
$$

by $(t, y)$ where $t$ represents the geodesic distance from $M_{0}$. Since the geodesics are orthogonal to $M_{t}$ we have, in (1.4), $a^{1, j}=0, j=2, \ldots, m$, and $a^{11}=1$. The operator $E_{k}$, therefore, is of the form

$$
E_{K} u=\frac{-1}{\sqrt{a}} \frac{\partial}{\partial t} \zeta^{2} \sqrt{a} \frac{\partial u}{\partial t}-\frac{1}{\sqrt{a}} \frac{\partial}{\partial y_{i}} \zeta^{2} \sqrt{a} a^{i j} \frac{\partial u}{\partial y_{j}}+K u=f .
$$

Since $k \geqslant 2$, each of the second derivatives makes sense as an $L_{2}$ strong derivative when $t>0$. It follows from standard theorems that

$$
\int_{M_{t}} u^{2}(t, y) \sqrt{a} d y, \quad \int_{M_{t}} u_{t}^{2} \sqrt{a} d y, \quad \int_{M_{t}}\left(\frac{\partial u}{\partial y_{i}}\right)^{2} \sqrt{a} d y
$$

exist and are continuous function of $t$. By solving for $u_{t t}$ in (5.4) and substituting in

$$
\int_{M_{t}} u_{t} u \sqrt{a} d y
$$

we may deduce, by integration by parts and (5.4), that (5.5) is also a continuous function to $t$. It follows from (5.4) and (5.5) that

$$
v(t)=\int_{M_{t}}[(t, y)]^{2} \sqrt{a} d y
$$

is a class $C^{2}$ for $0<t<\delta$. After multiplying (5.4) by $t^{2} u / \zeta^{2}$, using the identity

$$
u_{t t} u=\frac{1}{2}\left(u^{2}\right)_{t t}-u_{r}^{2},
$$

integrating over $M_{t}$, and integrating by parts in the manifold $M_{t}$, we find that the function $v$ defined by (5.6) satisfies

$$
\begin{aligned}
-\frac{t^{2}}{2} v_{t t}+K \int_{M_{t}} t^{2} \frac{u^{2}}{\zeta^{2}} \sqrt{a} d y= & \int_{M_{t}}\left[-t^{2}\left(\operatorname{grad}_{a} u\right)^{2}+\frac{t^{2}}{\zeta^{2}} f u\right] \sqrt{a} d y \\
& +\int_{M_{t}} t^{2}\left[\frac{A(y, t)}{\zeta} u_{t} u\right] \sqrt{a} d y
\end{aligned}
$$

where $A$ is a bounded function. There exists a constant $b>0$ such that

$$
1 / b<t / \zeta<b
$$

Hence, if we rewrite the left side of (5.7) as

$$
-\frac{t^{2}}{2} v_{t t}+\frac{K}{2 b^{2}} \int u^{2} \sqrt{a} d y+K \int\left(\frac{t^{2}}{\zeta^{2}}-\frac{1}{2 b^{2}}\right) u^{2} \sqrt{a} d y
$$


and bring the third term on the right in (5.9) over to the right side of (5.7), we may deduce that, for all sufficiently large $K, u$ sastisfies an equation of the form

$$
-t^{2} v_{t t}+\Lambda v=F(t), \quad \Lambda=\frac{K}{2 b^{2}},
$$

where $F$ is absolutely integrable on $(0, \delta)$ and has an upper bound

$$
F(t) \leqslant \text { const } \int_{M_{t}}|f(t, y)|^{2} \sqrt{a} d y
$$

hence

$$
\int_{0}^{\delta} F(t) d t<\text { const } \int_{M_{t}}|f(t, y)|^{2} \sqrt{y} d y .
$$

In particular, if $f \in C_{0}^{\infty}$ the right side of (5.12) is zero. We are thus led to the study of positive solutions of (5.10). We shall show that if $F$ is absolutely integrable and has an upper bound which tends to zero sufficiently fast, then positive solutions also tend to zero.

THEOREM 5.1. Let $v$ be a positive solution of (5.10) where

$$
\int_{0}^{\delta} \mid F(t) d t<\infty \text { and } \int_{0}^{\delta} v(t) d t<\infty .
$$

Let $\nu$ and $\mu$ be the positive and negative roots of

$$
\lambda(\lambda-1)-\Lambda=0
$$

where $\Lambda>2$ so that $\mu<-1$. If, for some $\rho>\nu$,

$$
F(t)<c t^{\rho}
$$

then there exists const $\alpha$ such that

$$
v(t)<\alpha t^{\nu}
$$

Proof. For $\delta>t>0$, the solution of (5.10) is

$$
v(t)=\frac{1}{(\nu-\mu)} \int_{t}^{\delta} \frac{t^{\nu}}{s^{\nu+1}} f(s) d s-\frac{1}{(\nu-\mu)} \int_{t}^{\delta} \frac{t^{\mu} F(s)}{s^{u+1}} d s+a t^{\nu}+b t^{\mu}
$$

where $a$ and $b$ are constants. After integrating both sides of (5.17) from $\tau$ to $\delta$ we obtain

$$
\begin{aligned}
\int_{\tau}^{\delta} v(t) d t= & \frac{a \delta^{\nu+1}}{\nu+1}+\frac{b \delta^{\mu+1}}{\mu+1}-\frac{1}{(\nu+1)(\mu+1)} \int_{\tau}^{\delta} F(s) d s \\
& +\frac{t^{\mu+1}}{\mu+1}\left[\frac{1}{(\nu-\mu)} \int_{\tau}^{\delta} \frac{F(s)}{s^{\mu+1}} d s-b\right]-\frac{\tau^{\nu+1}}{\nu+1}\left[\int_{\tau}^{\delta} \frac{F(s)}{s^{\mu+1}} d s-a\right] .
\end{aligned}
$$

Since $\nu>0,(\tau / s)^{\nu+1} \leqslant 1$ when $\tau \leqslant s \leqslant \delta$. This, together with (5.13), shows that all of the terms, with the possible exception of the fourth on the right side of the equality sign, are uniformly bounded for $0<\tau<\delta$. Hence, we must have

$$
b=\frac{1}{(\nu-\mu)} \int_{0}^{\delta} \frac{F(s)}{s^{\mu+1}} d s,
$$


the existence of the integral being insured by (5.10) and the fact that $\mu<-1$. After putting (5.19) into (5.17) we find that

$$
v(t)=a t^{\nu}+\frac{1}{(\nu-\mu)} \int_{t}^{\delta} \frac{t^{\nu}}{s^{\nu+1}} F(s) d s+\frac{1}{(\nu-\mu)} \int_{0}^{t} \frac{t^{\mu}}{s^{\mu+1}} F(s) d s .
$$

Since $\nu+\mu=1$, the proof follows by substituting the upper bound (5.15) into the right side of (5.20). Note that if $\rho=\nu$ one obtains only the bound $v(t) \leqslant$ const $t^{\nu} \log 1 / t$ and if $\rho<\nu$ the upper bound $v(t) \leqslant$ const $t^{\rho}$ is valid. However, in our application to degenerate elliptic equations we are able to obtain the upper bound (5.11) only for sufficiently large values of $K$.

THEOREM 5.2. Let $\Omega$ be a compact domain whose boundary is of class $C^{k}, k \geqslant 2$, and let $E_{K}$ be defined by (1.3) and satisfy the hypothesis of Theorem 4.1. Then, if $u$ is a solution of $E_{K} u=f \in C_{0}^{\infty}(\Omega)$, then for $|\alpha| \leqslant k-2$ the integrals

$$
\int_{M_{t}}\left|D^{\alpha} u(y, t)\right|^{2} \sqrt{a} d y
$$

can be made to vanish to any order as $t \rightarrow 0$ by taking $K$ sufficiently large. Moreover, if $v$ has $k-1$ strong derivatives in $\Omega$, then, for $\leqslant k-1$,

$$
\int_{\Omega} D^{\alpha} u v \sqrt{a} d x=(-1)^{\alpha} \int_{\Omega} u D \sqrt{\alpha} v d x
$$

Proof. When $k=2$, the proof follows from Theorem 5.1 and the fact that the bound (5.11) is valid for sufficiently large $K$. When $k>2$, the proof follows from successive application of Lemma 4.2. The integration by parts formula (5.22) is a consequence of the fact that (5.21) implies that the boundary integral, arising from integration by parts, vanishes.

We have been unable to prove the vanishing of the integrals (5.21) when $\alpha=k-1$ or $k$. The following theorem serves as a satisfactory substitute for our purposes.

THEOREM 5.3. Under the hypothesis of Theorem 5.2, then for any $\rho$ the integrals

$$
\int_{0}^{\delta} \int_{M_{t}} \frac{\left|D^{\alpha} u(y, t)\right|^{2}}{t^{\rho}} \sqrt{a} d y d t, \quad|\alpha|=k-1 \text { or } k,
$$

can be made finite by taking $K$ sufficiently large.

The proof if the estimates (5.21) when $\alpha \leqslant k-2$ depends on the fact that the upper bound (5.11) is a consequence of the inequality

$$
F(t)=-\int_{M_{t}} t^{2}(\operatorname{grad} u)^{2} \sqrt{a} d y+G(t)
$$

where $G$ is bounded by the right side of (5.11). Having obtained the bound (5.16) for the nonnegative term $v$, we may put (5.24) into (5.20) to obtain

$$
\int_{t}^{\delta} \int_{M_{t}} \frac{t^{\nu}}{s^{\nu-1}}|\operatorname{grad} u|^{2} \sqrt{a} d y d s+\int_{0}^{t} \int_{M_{t}} \frac{t^{\mu}}{s^{\mu+1}}|\operatorname{grad} u|^{2} \sqrt{a} d y d t \leqslant c t^{\nu}
$$


for some positive constant $c$. By ignoring the second term on the left in (5.25) we then obtain

$$
\int_{t}^{\delta} \int_{M} \frac{(\operatorname{grad} u)^{2}}{t^{\nu-1}} \sqrt{a} d y d s \leqslant c
$$

for any $t \in[0, \delta]$. By letting $t \rightarrow 0$ we obtain the desired result when $k=2$ and $|\alpha|=1$. The case $k=2,|\alpha|=2$, now follows from Lemma 3.6. The proof for general $k$ follows by using the arguments in the proof of Lemma 4.2 .

We are now in a position to complete the proof of Theorem 3.5. Consider first the case $m \geqslant 2$. Then for $\varphi \in \mathcal{H}_{m, k}$,

$$
\left\|E_{K} u\right\|_{-m, k} \geqslant \frac{\left|\left\langle E_{k} u, \varphi\right\rangle\right|}{\left\|E_{k} \varphi\right\|_{m-2, k}}
$$

since $\left\|E_{k} \varphi\right\|_{m-2, k}$ and $|u|_{m, k}$ are equivalent when $k \geqslant 0$. By Theorems 5.2 and 5.3, for $\psi \in C_{0}^{\infty}$ and $K$ sufficiently large, $\exists \varphi \in \mathfrak{H}_{m, k}$ such that $E_{K} \varphi=\psi$.

It follows from (5.27) that

$$
\left\|E_{K} u\right\|_{-m, k} \geqslant \frac{|\langle u, \psi\rangle|}{\|\psi\|_{m} \quad 2, k}
$$

for each $\psi \in C_{c}^{\infty}$. Hence,

$$
\left\|E_{K} u\right\|_{-m, k} \geqslant\|u\|_{-m+2, k}
$$

when $m$ is an integer $\geqslant 2$. When $m=1$,

$$
\left\|E_{K} u\right\|_{-1, k} \geqslant \frac{\left|\left\langle E_{k} u, \varphi\right\rangle\right|}{\|\varphi\|_{1, k}}
$$

for each $\varphi \in \mathfrak{H}_{1, k}$. By integrating by parts in the principal part of $E_{h}$ and choosing a sequence of $\varphi$ 's converging to $u$ in $\mathcal{H}_{1, k}$, we obtain

$$
\left\|E_{K} u\right\|_{-1, k} \geqslant\|u\|_{1, k} \text {. }
$$

Hence, (5.29) is valid for all positive integers $m$. The opposite inequality follows from Theorem 3.2 and 3.3.

6. Application to the Dirichlet equation for strongly uniformly elliptic equations. Let

$$
L=\sum_{|\alpha| \cdot|\beta| \leqslant m} D^{\alpha} a_{\alpha \beta} D
$$

be a uniformly strongly elliptic operator of degree $2 m$ in a domain $\Omega$ which is compact and of class $C^{m+\nu}$. The coefficient $a_{\alpha \beta}$ with $|\alpha|+|\beta|<2 m$ is assumed to satisfy

$$
\left|D^{\gamma} a_{\alpha \beta}(x)\right| \leqslant M_{\gamma, \alpha, \beta} \rho^{|\alpha|+|\beta|-\gamma-2 m+\varepsilon}, \quad|\gamma| \leqslant \nu,
$$

where $\rho$ is the distance from $x$ to the boundary of $\Omega$. The equivalence of the norms $\|u\|_{m, m}$ and the classical Sobolev $m$-norm proved in [11], together with the classical treatment of the Dirichlet Problem, could be used to prove when $\nu=0$ and $\operatorname{Re} \lambda$ is sufficiently large there exists a unique solution $u \in \mathcal{H}_{m, m}$ of

$$
(L+\lambda) u=f
$$


with generalized zero Dirichlet data for $f \in \mathcal{H}_{-m,-m}$. This improves the classical result by allowing both $f$ and certain of the coefficients to grow at the boundary. The conclusion that when $f=\mathcal{K}_{0,0}$ then $u \in \mathcal{H}^{2 m}$ could not be deduced without relaxing the singularities of the coefficients at the boundary. We shall prove in this section that under condition (6.2) there is a unique solution $u \in \mathscr{H}_{m+\nu, m}$ when (6.2) is satisfied and $f \in \mathcal{H}_{-(m-\nu),-m}$, thus allowing the derivatives of order greater then $m$ to grow at the boundary.

Let us use the degenerate operator $E_{K}$ with $a_{i j}=\delta_{i j}$, and consider the form

$$
\operatorname{Re} \int E_{K}^{\nu} L \varphi \bar{\varphi} d x
$$

It follows from (3.8) and (3.9) that (6.4) may be written

$$
\operatorname{Re}(-1)^{m} \int(-1)^{\nu}(L+\lambda) \zeta^{\nu} \varphi \overline{\zeta^{\nu} \varphi} d x+\operatorname{Re} K^{\nu}(-1)^{m} \int(L+\lambda) \varphi \cdot \bar{\varphi} d x
$$

plus terms which, via the interpolation inequality, can be dominated by

$$
\|u\|_{m+\nu, m+\nu}^{2}+\|u\|_{m}^{2} \text {. }
$$

The Gårding inequality gives, for $u \in \bigodot_{0}^{\infty}(\Omega)$,

$$
\operatorname{Re}(-1)^{m} \int(-1)^{\nu}(L+\lambda) \zeta^{\nu} \varphi \cdot \overline{\zeta^{\nu} \varphi} d x \geqslant\left\|\zeta^{\nu} \varphi\right\|_{m+\nu, m+\nu}^{2}
$$

and

$$
\operatorname{Re}(-1)^{m} \int(L+\lambda) \varphi \bar{\varphi} d x \geqslant\|\varphi\|_{m, m}
$$

provided that $\operatorname{Re} \lambda$ is sufficiently large. Since by Theorem 3.1,

$$
\left\|\zeta^{\nu} \varphi\right\|_{m+\nu, m+\nu} \sim\|\varphi\|_{m+\nu, m},
$$

it follows from (6.5), (6.6), and the interpolation inequality that for fixed sufficiently large $\operatorname{Re} \lambda$ and all sufficiently large $K$ that

$$
\operatorname{Re} \int_{\Omega} E_{k}^{\nu} L \varphi \varphi d x \geqslant\|\varphi\|_{m+\nu, m}^{2}
$$

for all $u \in C_{0}^{\infty}(\Omega)$. By the generalized Cauchy-Schwarz inequality (2.4) it follows that

$$
\left|\operatorname{Re} \int E_{k}^{\nu} L \varphi \varphi d x\right| \leqslant\left\|E_{k}^{\nu} L \varphi\right\|_{-m-\nu,-m}\|\varphi\|_{m+\nu, m} .
$$

Hence by (6.9) and (6.10),

$$
\left\|E_{k}^{\nu} L \varphi\right\|_{-m-\nu, m} \leqslant\|f\|_{\nu-m,-m} .
$$

Hence by (6.11) and (6.10), if $f \in \mathcal{K}_{\nu-m, m}$ the linear functional

$$
L^{*} E_{k}^{\nu} \varphi \rightarrow\left\langle E_{k}^{\nu} f, \varphi\right\rangle
$$

is bounded on a closed linear subspace of $\mathcal{H}_{-m-\nu,-m}$. It follows from the HahnBanach Theorem that there exists a $u_{\nu} \in \mathcal{H}_{m+\nu, m}$ such that

$$
\left\langle u_{\nu}, L^{*} E_{k}^{\nu} \varphi\right\rangle=\left\langle E_{k}^{\nu} f, \varphi\right\rangle
$$


for all $\varphi \in C_{0}^{\infty}(\Omega)$. For $\psi \in C_{0}^{\infty}(\Omega)$ we now take $\varphi$ to be a solution of

$$
E_{k} \varphi=\psi \text {. }
$$

As a consequence of Theorems 5.2 and 5.3, for $K$ sufficiently large, $\varphi \in \mathcal{H}_{m+\nu, \nu}$, and we may integrate by parts to deduce that

$$
\left\langle u_{2} L^{*} E_{k}^{\nu-1} \psi\right\rangle=\left\langle E_{k}^{\nu-1}, \psi\right\rangle, \quad \psi \in C_{0}^{\infty}(\Omega),
$$

that is, $u_{\nu}$ is a weak solution of

$$
E_{k}^{\nu-1} L u_{\nu}=E_{k}^{\nu-1} f .
$$

By induction, since for $j=0$ the solution is unique, we have $u_{j}=u_{0}$ for $j=0,1, \ldots, \nu$. This completes the proof.

7. Application to the Cauchy Problem for strongly hyperbolic equations. In this section we shall show how the degenerate elliptic operator $E_{K}$ can be used to obtain existence, uniqueness and regularity for solutions with zero Cauchy data of an $m$ th degree strongly hyperbolic equation,

$$
P\left(D_{t}, D_{x}\right) u=\sum_{k+|\alpha| \leqslant m} \mathbb{Q}_{k \alpha}(t, x) D_{t}^{k} D_{x}^{\alpha} u=f(t, x),
$$

defined for $(t, x)$ in the strip $0 \leqslant t \leqslant \delta, x \in R_{n}$. We shall use the weighted norms and shall exploit the fact that we can use weights which vanish to different orders on the top and bottom of the strip. The notation

$$
\|u\|_{m, k_{1}, k_{2}}
$$

means the norm (1.2) with $k=k_{1}$ along $t=0$ and $k=k_{2}$ on $t=\delta$. For example, we could use the weight $(\delta-t)^{k_{2}} t^{k_{1}}$.

The condition of hyperbolicity is that the principal part $P_{m}$ of $P$ satisfies $\mathscr{Q}_{m 0} \neq 0$,

$$
P_{m}(t, x, \tau, \xi)=Q_{m 0}(t, x) \prod_{j=1}\left(\tau-\tau_{j}(t, x, \xi)\right)
$$

where for $\xi \neq 0$ and for all $t, x$ the roots $\tau_{j}$ are real and distinct. Let us assume at first that $P_{m}$ has constant coefficients and consider the bilinear form

$$
\operatorname{Re} \int_{0<t<\delta}(\delta-t)^{2 m-1} P_{m}\left(D_{t}, D_{x}\right) u \frac{\partial}{\partial \tau} \overline{P_{m}\left(D_{t}, D_{x}\right) u} d x d \tau
$$

for $u \in C_{0}^{\infty}\left\{(0, \delta] \times R_{n}\right\}$. After taking Fourier transforms with respect to the spatial variables and applying the Parseval identity, we have

$$
\operatorname{Re} \int(\delta-t)^{2 m-1} P_{m}\left(D_{t}, \xi\right) \hat{u}(\tau, \xi) \overline{\frac{\partial}{\partial \tau} P_{m}\left(D_{t}, \xi\right) \hat{u}(\tau, \xi)} d \xi d \tau .
$$

Assuming, as we may, that $\mathbb{Q}_{m 0}=1$, it follows from (7.3) that (7.5) may be written

$$
\frac{1}{2} \operatorname{Re} \int_{0<t<\delta}(\delta-t)^{2 m-1} \sum_{j=1}^{m} \frac{\partial}{\partial \tau}\left|L_{j} \hat{u}\right|^{2} d \xi d t
$$

where the $L_{j}$ are the Lagrange interpolation polynomials

$$
L_{j}=\frac{P_{m}}{\tau-\tau_{j}} .
$$


After integrating the parts and using the fact that the Lagrange interpolation polynomials span the polynomials of degree $m-1$, another application of the Parseval identity shows that the expression (7.6) is bounded from below by

$$
\text { const } \int_{0<t<\delta}(\delta-t)^{2(m-1)} \sum_{|\alpha|=m-1}\left|D^{\alpha} u(t, x)\right|^{2} d x d t
$$

for all $u \in C_{0}^{\infty}\left[(0, \delta] \times R_{n}\right]$. The quantity (7.8) is the leading term in the norm $\|u\|_{m-1, m-1,0}$. Thus, to complete the proof of the a priori estimate in the case of constant coefficients, we need an inequality on one-dimensional calculus,

$$
\int_{0}^{\delta} \frac{(\delta-t)^{2 a}}{t^{2 b}}|u(t)|^{2} d t \leqslant \frac{C_{a b}}{\delta^{2}} \int_{0}^{\delta} \frac{(\delta-t)^{2(a+1)}}{t^{2(b-1)}}\left|u^{\prime}(t)\right|^{2} d t,
$$

valid for $b \geqslant 1, a \geqslant 0$ and $u \in C^{\prime}$ and vanishing near zero. To prove (7.9), we write

$$
|u(t)|^{2}=2 \operatorname{Re} \int_{0}^{t} u(s) \overline{u^{\prime}(s)} d s .
$$

After multiplying both sides of (7.9) by $(\delta-t)^{2 a} / t^{2 b}$ and integrating from 0 to $\delta$ we interchange orders of integration to obtain

$$
\int_{0}^{\delta} \frac{(\delta-t)^{2 a}}{t^{2 b}}|u(t)|^{2} d t \leqslant 2 \int_{0}^{\delta} \int_{s}^{\delta} \frac{(\delta-t)^{2 a}}{t^{2 b}} d t \mid u(s) \| u^{\prime}(s) d s .
$$

Since $a \geqslant 0$ and $b \geqslant 1$, it is easily verified that

$$
\int_{s}^{\delta} \frac{(\delta-t)^{2 a}}{t^{2 b}} \leqslant \text { const } \frac{(\delta-s)^{2 a+1}}{s^{2 b-1}} .
$$

An application of the Schwarz inequality then shows the inequality (7.9) to be valid for some constant. Since, by making a change of variable, we may assume that $\delta=1$, the constant must have the indicated form. It follows from (7.4)-(7.9) that if $P_{m}$ is hyperbolic with constant coefficients, then

$$
\operatorname{Re} \int_{0<t<\delta}(\delta-t)^{2 m-1} P_{m} u \overline{\frac{\partial P_{m}}{\partial \tau}} u d x d t \geqslant C\|u\|_{m-1, m-1,0}^{2}
$$

for some positive constant $C$ and all $u \in C_{0}^{\infty}\left[(0, \delta] \times R_{n}\right]$.

Assume next that $P_{m}$ has variable coefficients which are of class $C^{\prime}$ and uniformly continuous. It follows from Hörmander [4, Lemma 8.2.2, p. 189] that

$$
\begin{aligned}
& \operatorname{Re} \int_{0<t<\delta}(\delta-t)^{2 m-1} P_{m} u \overline{\frac{\partial P_{m}}{\partial \tau}} u d x d t \\
& \quad=\frac{(2 m-1)}{2} \operatorname{Re} \int_{0<t<\delta}(\delta-t)^{2(m-1)}\left\{P_{m} u \overline{\frac{\partial^{2} P_{m}}{\partial \tau^{2}} u}-\left|\frac{\partial^{2} P_{m}}{\partial \tau^{2}}\right|^{2}\right\} d x d t
\end{aligned}
$$

plus a term bounded by

$$
C \delta\|u\|_{m-1, m-1,0},
$$

the constant depending on the bound for the first derivatives of the coefficients. By choosing a partition of unity $\Sigma \varphi_{j}^{2}$ with support sufficiently small relative to the 
modulus of continuity of the coefficients, we arrive at an expression with the right side of (7.14) being a sum with $u$ replaced by $\varphi_{j} u$ and coefficients frozen at a point in the support of $\varphi_{j}$. This introduces another error which can be bounded by (7.15). By using (7.14) in reverse and applying the inequality (7.15) for constant coefficients, one then arrives at the inequality (7.13) with $P_{m}$ having variable coefficients and perturbed by an expression of the form (7.15). Hence for $\delta$ sufficiently small, the inequality (7.13) is valid for operators with variable coefficients having the indicated regularity.

The presence of the negative powers of $t$ in the coefficients of the derivatives of order less than $2(m-1)$ in the norm $\|u\|_{m-1, m-1,0}$ allows us to improve the classical result by allowing singularities in the lower order coefficients.

THEOREM 7.1. Let $P$ be hyperbolic of degree $m$ with respect to the direction $(1,0, \ldots, 0)$ in the strip $(0, \delta) \times R_{n}$. If the coefficients of the principal part $P_{m}$ are uniformly continuous and have uniformly bounded derivatives and if the lower order coefficients $\mathbb{Q}_{k, \alpha}$ in (7.1) satisfy, for some $\varepsilon>0$,

$$
\left|\hat{\psi}_{n, \alpha}\right| \leqslant M_{k}|\alpha|^{t^{-\rho}}, \quad \rho=m-k-|\alpha|-\varepsilon,
$$

then for sufficiently small $\delta$ and all $u \in C_{0}^{\infty}\left[(0, \delta] \times R_{n}\right]$ we have

$$
\operatorname{Re} \int_{0<t<\delta}(\delta-t)^{2 m-1} P u \overline{\frac{\partial P_{m}}{\partial \tau}} u d x d t \geqslant C\|u\|_{m-1, m-1,0}^{2} .
$$

and if $P^{*}$ is the formal adjoint of $P$

$$
\operatorname{Re} \int_{0<t<\delta} t^{2 m-1} P^{*} u \overline{\frac{\partial P_{m}}{\partial \tau}} u d x d t \geqslant C\|u\|_{m-1 . m-1.0}
$$

for all $u \in C_{0}^{\infty}\left\{[0, \delta) \times R_{n}\right\}$.

Inequality (7.17) was proved preceding its statement, and (7.18) follows by replacing $t$ by $\delta-t$ and requiring that $u$ vanish near the top of the strip.

In order to study regularity, we shall use the degenerate operator

$$
E_{K}=-\frac{\partial}{\partial t} \zeta^{2} \frac{\partial}{\partial t}-\alpha^{2} \zeta^{2} \Delta_{x}+K u
$$

where $\zeta=t(\delta-t)$. The proof will be a consequence of Gårding's inequality by virtue of the positivity of a quadratic form given by the following lemma.

Lemma 7.2. Let $P(\tau)=\prod_{j=1}^{m}\left(\tau-\tau_{j}\right)$ be a polynomial of degree $m$ with only real roots and let $A(\tau)=\tau^{2}+\alpha^{2}$. Then for all sufficiently large $\alpha$, the form

$$
\left(A^{\nu} P\right)^{\prime} P^{\prime}-A^{\nu} P P^{\prime \prime}
$$

is positive.

Proof. By direct computation one finds that the expression (7.20) is equal to

$$
E^{\nu} P^{2} \sum_{j=1}^{m} \frac{(2 \nu+1) \tau^{2}-2 \nu \tau_{j} \tau+\alpha^{2}}{\left(\tau-\tau_{j}\right)^{2}\left(\tau^{2}+\alpha^{2}\right)}
$$

from which the result follows immediately. 
Now let $E_{k}$ be the degenerate elliptic operator based on the quadratic form

$$
\tau^{2}+\alpha^{2}|\xi|^{2}
$$

where $\alpha$ is so large that the form (7.20) is positive definite. Another application of Hörmander [4, Lemma 8.2.2, p. 189] gives

$$
\begin{aligned}
2 \operatorname{Re} & \int_{0<t<\delta}(\delta-t)^{2 m-1}(-1)^{\nu}\left(\frac{\partial^{2}}{\partial t^{2}}+\alpha^{2} \Delta_{x}\right)^{\nu} P_{m} u \frac{\partial P_{m}}{\partial \tau} u d x d t \\
& =(2 m-1) \operatorname{Re} \int_{0<t<\delta}(\delta-t)^{2 m-2}\left[\frac{\partial}{\partial \tau}\left(A^{\nu} P_{m}\right) u \frac{\partial P_{m}}{\partial \tau} u-A^{\nu} P_{m} u \frac{\partial^{2} P_{m}}{\partial \tau^{2}} u\right] d x d t
\end{aligned}
$$

It follows from Lemma 7.2, (7.4) and Gårding's inequality that the right side of (7.23) is bounded from below by

$$
\|u\|_{m+\nu-1, m-1,0}
$$

for all $u \in C_{0}^{\infty}\left[(0, \delta) \times R_{n}\right]$ and hence for $u \in C^{\infty}\left[(0, \delta] \times R_{n}\right]$ by the equivalence theorem proved in [11]. By replacing $u$ by $\zeta^{\nu} u$ in (7.23), choosing $\delta$ sufficiently small and $K$ sufficiently large, we find that

$$
\operatorname{Re} \int_{0<1<\delta}(\delta-t)^{2 m-1} E_{K}^{\nu} u P u \frac{\overline{\partial P_{m} u}}{\partial \tau} d x d t \geqslant \varepsilon\|u\|_{m+\nu-1, m-1,0}^{2}
$$

provided that the coefficients of $P$ satisfy the additional regularity condition

$$
\left|D^{\beta} \mathbb{Q}_{n, \alpha}\right| \leqslant M_{k, \alpha, \beta} t^{-\rho}, \quad \rho=m-k-|\alpha|+|\beta|-\varepsilon,
$$

when $|\beta| \leqslant \nu$.

It now follows from (7.23) and the generalized Cauchy-Schwarz inequality that

$$
\|u\|_{m+\nu-1, m-1,0} \leqslant C\left\|E_{k}^{\nu} P u\right\|_{-\nu, 0,-m}
$$

for all $u \in C_{0}^{\infty}\left[(0, \delta] \times R_{n}\right]$. Similarly,

$$
\|\varphi\|_{m+\nu-1,0, m-1} \leqslant C\left\|P^{*} E_{k}^{\nu} \varphi\right\|_{-\nu,-m, 0}
$$

for all $\varphi \in C_{0}^{\infty}\left[(0, \delta) \times R_{n}\right]$.

It follows from (7.26) and the Hahn-Banach Theorem that when $f \in \mathcal{H}_{1+\nu-m, 0,1-m}$ the map

$$
P^{*} E_{K}^{\nu} \varphi \rightarrow\left\langle E_{k}^{\nu} f, \varphi\right\rangle, \quad \varphi \in C_{0}^{\alpha}\left[[0, \delta) \times R_{n}\right],
$$

is expressible as

$$
\left\langle u_{\nu}, P^{*} E_{K}^{\nu} \varphi\right\rangle=\left\langle E_{K}^{\nu} f, \varphi\right\rangle
$$

for some $u_{\nu}=\mathcal{H}_{\nu, m, 0}$. Since for $\psi \in C_{0}^{\infty}$ we may always, by taking $K$ sufficiently large, find a test function $\varphi$ such that $E_{k} \varphi=\psi$, it follows by induction that

$$
\left\langle u_{\nu}, P^{*} E_{k}^{j} \psi\right\rangle=\left\langle E_{k}^{j} f, \psi\right\rangle, \quad j=0,1, \ldots, \nu,
$$

for all $\psi \in C_{0}^{\infty}\left[[0, \delta] \times R_{n}\right]$. That is, $u_{\nu}$ is a weak solution in $\mathcal{H}_{\nu, m, 0}$ of

$$
E_{K}^{j} P u=E_{K}^{j} f
$$


for each $j=0,1, \ldots, \nu$. If $\nu=m$, we may invoke (7.26) to deduce that $u_{0}$ is unique. But, since $u_{\nu} \in \mathcal{H}_{m, m, 0}$ is also a solution, we must have $u_{0} \in \mathcal{H}_{m, m, 0}$. Hence, we have proved the following theorem.

THEOREM 7.3. If $P$ satisfies the conditions of Theorem 7.1 and (7.24) with $\nu=m+r$, then for $f \in \mathcal{H}_{r+1,0,1-m}$ there exists a unique solution $u \in \mathcal{H}_{m+r, m, 0}$ of the zero Cauchy data problem for $\mathrm{Pu}=f$.

8. Improved estimates at the boundary. The results of the two preceding sections improve the corresponding classical interior regularity results by allowing the coefficients and their derivatives to grow at the boundary. The norm used in $\$ 7$ precisely characterizes the notion of generalized zero Cauchy data. The hypothesis is best possible for a norm which failed to distinguish between time and spatial derivatives. However, if we distinguish the roles of the time and spatial derivatives, we find that we have postulated one more time derivative of the forcing function than necessary, see Hörmander [4]. This defect could be taken care of by introducing, in place of $E_{K}$, a degenerate operator depending only on the spatial derivatives.

For the Dirichlet Problem for elliptic equations, since the forcing term is allowed to grow at the boundary which in turn allows sufficiently high order derivatives to grow at the boundary, our theorems of $\$ 6$ neither imply nor are implied by the classical boundary solvability results in the spaces $\mathcal{H}_{r} \cap \mathcal{K}_{s}$. In this section we show how modifications of the degenerate operator $E_{K}$ can be used to obtain more refined results from which the classical estimates can be deduced.

In terms of the geodesic normal-tangent coordinate system described in $\S 5$, we replace the operator $E_{K}$ in a neighborhood of the boundary by

$$
A_{K} u=-\frac{1}{\sqrt{a}} \frac{\partial}{\partial t} \sqrt{a} t^{2} \frac{\partial u}{\partial t}-\frac{1}{\sqrt{a}} \frac{\partial}{\partial y_{i}} \sqrt{a} a^{i j} \frac{\partial u}{\partial y_{j}}+K u,
$$

say for $0 \leqslant t \leqslant \delta / 2$. By smoothening off $t^{2}$ to 1 between $\delta / 2$ and $\delta$, and defining $A_{K}$ to be the ordinary Laplace-Beltrami operator when $t \geqslant \delta$, we may assume that $A_{K}$ is globally defined. We denote by $\Omega_{\delta}$ the set of points whose distance from the boundary is less than $\delta$ and define

$$
\|u\| \|_{m, k}^{2}=\sum_{|\alpha|+j \leqslant m} \int_{\Omega_{\delta}} t^{2(j-k)}\left|D_{t}^{j} D_{y}^{\alpha} u\right|^{2} \sqrt{a} d y d t+\sum_{|\alpha| \leqslant m} \int_{\Omega_{-}-\Omega_{\delta}}\left|D^{\alpha} u\right|^{2} d x
$$

Since we have used $\zeta=t$, the geodesic distance from the boundary, it is easily proved as in [11] that the completion of the $C_{0}^{\infty}$ functions is the same as the $C^{\infty}$ functions with finite norm. Note, however, that for a $\zeta$ which depends on both $t$ and $y$, the theorem would, in general, be false for the norm (8.2).

The question of the extent to which the highest order terms characterize the norm (8.2) is answered by the following lemma.

LEMMA 8.1. If either

$$
a>1 / 2 \text { and } u \in C_{0}^{1}\left(0, t_{0}\right]
$$




$$
a<1 / 2 \text { and } u \in C_{0}^{1}\left[0, t_{0}\right)
$$

then

$$
\int_{0}^{t_{0}} t^{-2 a}|u(t)| d t \leqslant \frac{4}{(2 a-1)^{2}} \int_{0}^{t_{0}} t^{2(1-a)}\left|u^{\prime}(t)\right|^{2} d t .
$$

In particular, if $a \neq 1 / 2$ and $u \in C_{0}^{1}\left(t, t_{0}\right)$ then (1.5) is valid.

Proof. The inequality (8.5) was proved under condition (8.3) in [11]. To prove it under condition (8.4), write

$$
|u(t)|^{2}=-2 \operatorname{Re} \int_{t}^{t_{0}} u(s) \overline{u^{1}(s)} d s
$$

and proceed as in [11]. That it is impossible to find a constant $C>0$ such that

$$
\int_{0}^{t_{0}} t^{-1}|u(t)|^{2} d t \leqslant C \int_{0}^{t_{0}} t\left|u^{\prime}(t)\right|^{2} d t, u \in C_{0}^{1}\left(0, t_{0}\right)
$$

is seen by choosing $u=t^{\alpha}(1-t)$, computing the Rayleigh quotient corresponding to (8.7) and then letting $\alpha \rightarrow 0$. As a consequence of Lemma 8.1 we have the following lemma.

LEMMA 8.2. If $m \leqslant k$, then the contribution to the norm (8.2) from the boundary strip $0 \leqslant t \leqslant \delta$ is dominated by its highest order terms. If $k \geqslant m$ and $k \neq 1 / 2(\bmod 1)$ then the contribution to the norm (8.2) from the boundary strip $0 \leqslant t \leqslant \delta$ is bounded by its highest order terms plus

$$
\text { const } \int_{\delta / 2 \leqslant t \leqslant \delta}|u|^{2} \sqrt{a} d y d t .
$$

Proof. The first assertion is obvious. To prove the second we choose $\chi \in C^{\infty}\left[R_{1}\right]$ with $\chi=1,0 \leqslant t \leqslant \delta / 2$ and $\chi=0$ for $t \geqslant \delta$. The proof then follows by writing

$$
u=\chi u+(1-\chi) u
$$

and applying Lemma 8.1 and the standard interpolation inequalities.

The proofs in $\$ \S 3-5$ go through without change if the operator $E_{K}$ is replaced by $A_{K}$ and the double bar norms are replaced by the corresponding triple bar norms. The a priori inequalities of $\$ \S 6$ and 7 require slightly more delicate analysis since the normal and tangential derivatives are weighted differently. If one separates the terms in $\left[A_{K}\right]^{\nu}$ into purely normal, purely tangential and mixed derivatives, the positivity of the terms in $A_{K}^{\nu} P$ resulting from the purely normal and purely tangential derivatives in bilinear forms (6.5), (7.23) can be shown to be positive as a consequence of the a priori inequalities corresponding to the case $\nu=0$.

The mixed derivatives can be dominated by the above derivatives if the spacial part of $A_{K}$ is multiplied by a sufficiently large constant.

Let us denote by $\mathcal{H}_{m, k}^{t}$ the completion of $C_{0}^{\infty}(\Omega)$ under the norm $\|u\| \|_{m, k}$. We then obtain the following theorem.

THEOREM 8.3. Under the hypothesis of $\S 6$, if $f \in \mathcal{K}_{\nu-m,-m}^{t}$ there exists a unique solution $u$ of $(L+\lambda) u=f$ with $u \in \mathcal{H}_{m+\nu, m}^{t}$. 
The above theorem is precisely the theorem on regularity to the boundary of tangential derivatives, see Browder [1] and Nirenberg [8]. The proof of strong differentiability to the boundary then takes the following form.

THEOREM 8.4. If the hypothesis of Theorem 8.3 is replaced by $f \in \mathcal{H}_{\nu-m, \nu-m}^{\prime}$ then $u$ has strong derivatives to the boundary up to order $m+\nu$.

Theorems 8.3 and 8.4 are both special cases of Theorem 9.1.

9. Normal derivatives at the boundary. Since Theorem 8.3 postulates $t^{m} f \in L_{2}$, in contrast to the assumption $f \in L_{2}$ used in the classical results, the conclusion cannot be improved without restricting the boundary behavior of $f$. In this section we shall show that replacing the assumption $t^{m} f \in L_{2}$ by $t^{m-\mu} f \in L_{2}$, we may conclude that the derivatives $D_{t}^{j} D_{x}^{\alpha} u, j \geqslant m, j+|\alpha| \geqslant m$ satisfy

$$
t^{j-\mu-m} D_{t}^{j} D_{x}^{j} u \in L_{2} \text {. }
$$

Let us introduce the weighted norm

$$
\begin{aligned}
{[u]_{r, m+\mu}^{2}=} & \sum_{r \geqslant j+|\alpha| \geqslant m+\mu} \int t^{2(j-\mu-m)}\left|D_{t}^{j} D_{x}^{\alpha} u\right|^{2} d t d x \\
& +\sum_{|\alpha| \leqslant m+\mu} \int\left|D^{\alpha} u\right|^{2} d x d t .
\end{aligned}
$$

Denote by $\overline{\mathcal{K}}_{r, m+\mu}$ the completion of $C^{\infty}$ functions under the norm (9.2).

We then obtain the following generalization of Theorems 8.3 and 8.4.

THEOREM 9.1. If, in addition to the hypothesis of Theorem 8.3, $f \in \mathcal{K}_{\nu-m, \mu-m}^{\prime}$, for some integer $\mu, 0<\mu<\nu$, then $(L+\lambda) u=f$ has a unique solution $u \in \mathcal{H}_{m+\nu, m}^{t} \cap$ $\overline{\mathcal{K}}_{m-\nu, m+\mu}$.

Proof. Since we are assuming that $\mu$ and $\nu$ are integers we may apply Theorem 8.3 without violating the exceptional case of $\frac{1}{2}$. Since $\mathcal{H}_{\nu-m, \mu-m}^{t} \subset \mathcal{H}_{\nu-m,-m}^{t}$ we may deduce, from Theorem 8.3, the existence of a solution $u \in \mathcal{H}_{m+\nu, m}^{t}$. Let us now rewrite the operator $L$ in the form

$$
L u=D_{t}^{m} M u+L^{\prime} u
$$

where $M$ is a differential operator of order $m$ and $L^{\prime}$ is a differential operator of order $2 m$ with no terms containing more than $m$ differentiations in the normal direction. The hypothesis that $f \in \mathscr{K}_{-m,-m}^{t}$ already implies that $L^{\prime} u \in \mathscr{H}_{1-m, 1-m}^{t}$ so the strengthened hypothesis $f \in \mathcal{H}_{\nu-m, 1-m}^{t}$ implies that $f-L^{\prime} u \in \mathcal{H}_{\nu-m, 1-m}$. Hence

$$
D_{t}^{m} M u \in \mathscr{K}_{\nu-m, 1-m} \text {. }
$$

It follows that

$$
M u \in \mathcal{H}_{\nu, 1} .
$$

We may now use Theorem 8.3 to interpolate between $\mathcal{K}_{\nu-m, 1-m}$ and $\mathcal{K}_{\nu, 1}$ and deduce that

$$
D_{t}^{j} M u \in \mathcal{K}_{1+j-m, 1+j-m}
$$

for $j=0,1, \ldots, m$. 
The ellipticity of $L$ implies that the coefficient of $D_{t}^{m}$ in $M u$ is nonzero. Hence, we may prove by finite induction that

$$
\int_{\Omega} t^{2(m+1-j)}\left|D_{t}^{m+1} u\right|^{2} d x d t<\infty
$$

for $j=0,1, \ldots, \nu$.

This completes the proof of Theorem 9.1 for the case $\mu=1$. The proof for $2<\mu \leqslant \nu$ follows by induction.

ACKNOWLEDGEMENT. This work was supported in part by the National Science Foundation grants NSF GP 7662, NSF GP 12182 and NSF MCS77-09237.

\section{BIBLIOGRAPHY}

1. F. E. Browder, On the regularity of solutions of elliptic differential equations, Comm. Pure Appl. Math. 9 (1956), 351-361.

2. G. Fichera, Linear elliptic differential systems and boundary value problems, Springer-Verlag, Berlin and New York, 1965.

3. K. O. Friedrichs, Symmetric positive linear differential equations, Comm. Pure Appl. Math. 11 (1958), 333-418.

4. L. Hörmander, Linear partial differential operators, Springer-Verlag, Berlin, 1963.

5. J. Kohn and L. Nirenberg, Degenerate elliptic parabolic equations of the second order, Comm. Pure Appl. Math. 20 (1967), 797-872.

6. P. D. Lax, On the Cauchy Problem for hyperbolic equations and the differentiability of solutions of elliptic equations, Comm. Pure Appl. Math. 8 (1955).

7. S. Mizohata, Unicite du prolongment des solution des equations elliptiques du quatrieme ordre, Proc. Japan Acad. 34 (1958), 687-692.

8. L. Nirenberg, Remarks on strongly elliptic equations, Comm. Pure Appl. Math. 8 (1955), 648-674.

9. O. Oleinik and E. V. Radkevič, Second order elliptic equations with nonnegative characteristic form, Plenum Press, New York, 1973.

10. O. Oleinik, The Cauchy Problem for hyperbolic equations of second order degenerating in a region and on its boundary, Dokl. Akad. Nauk SSSR 169 (1966), 525-528.

11. R. N. Pederson, An equivalent norm for the Sobolev space $\mathcal{H}_{m}$, J. Analyse Math. 36 (1979), 213-216.

12. On the Unique Continuation Theorem for certain second and fourth order elliptic equations, Comm. Pure Appl. Math. 11 (1958), 67-80.

13. L. Schwarz, Théorie des distributions. I, II, Hermann, Paris, 1950-1951.

Department of Mathematics, Carnegie-Mellon University, Pittsburgh, Pennsylvania 15213 